\title{
A cost-effective and eco-friendly way to dihydroxylate terminal and internal alkenes using hydrogen peroxide/formic acid
}

\author{
Chia-Wei Hsua \\ Trevor L. Dzwiniel*a \\ Krzysztof Z. Pupeka \\ ${ }^{a}$ Applied Materials Division, Argonne National Laboratory, \\ 9700 S. Cass Ave, Lemont, Illinois 60439, U.S.A. \\ * indicates the corresponding author. \\ tdzwiniel@anl.gov \\ Click here to insert a dedication
}

\author{
terminal or internal alkenes $\frac{1.05 \text { equiv. } \mathrm{H}_{2} \mathrm{O}_{2}}{1.06 \text { equiv. } \mathrm{HCOOH}} \underset{75^{\circ} \mathrm{C}, 6 \mathrm{~h}}{\longrightarrow}$ diols
}

No catalyst or base is needed!

Abstract In this report, we present a modified dihydroxylation procedure for terminal and internal alkenes under neat conditions using formic acid and hydrogen peroxide. Upon the in situ formation of performic acid, followed by the hydrolysis of hydroxy-formoxy compounds with excess water, we can obtain a series of diols without catalysts and bases.

Key words catalyst-free dihydroxylation of alkene; formic acid; hydrogen peroxide

Vicinal diols are paramount building blocks in fine chemical syntheses and medicinal chemistry. ${ }^{1}$ They are also common everyday substances such as ethylene glycol and propylene glycol, both well-known antifreeze chemicals. Propylene glycol is also applied in cosmetics ${ }^{2}$, food and pharmaceutical industry. ${ }^{3}$ In battery chemistry, vicinal diols are precursors of cyclic carbonates forming battery electrolytes such as ethyl carbonate (EC) ${ }^{4}$ and propylene carbonate (PC). ${ }^{5}$ To obtain vicinal diols, the dihydroxylation of alkenes has been broadly studied using tungsten $6 \mathrm{a}$, vanadium ${ }^{6 a}$, rhenium ${ }^{6 b}$, osmium ${ }^{6 c}$, etc. as the catalysts. However, the toxicity, cost and availability of these metals are highly concerned. Thus, a cost-effective and ecofriendly method will be valuable to the vicinal diol manufacture. Using a minimum amount of non-metal oxidizing agent is a plausible solution to provide a greener way to produce vicinal diols.

Organic peroxide compounds are commonly used to form epoxides from alkenes, followed by hydrolysis to produce diols with bases or acids-a two-step synthesis.6a For instance, metachloroperoxybenzoic acid $(m-\mathrm{CPBA})^{7}$, performic ${ }^{8,9}$ and peracetic acid $^{8}$ are widely utilized. Storage and waste control of organic peroxides emerge from the dihydroxylation of alkene on a large scale. In situ production of organic peroxide has been reported and applied to the dihydroxylation of alkenes. ${ }^{8,9}$ For instance,
Swern and his coworkers utilized formic acid and hydrogen peroxide to produce performic acid and oxidize the unsaturated fatty acids. They used sodium hydroxide to hydrolyze epoxides and hydroxy-formoxy species to obtain diols (Scheme 1). ${ }^{8}$ The other representative example is cyclohexene dihydroxylation ${ }^{9}$ with a similar protocol to Swern's. Formic acid and hydrogen peroxide formed performic acid in situ, followed by epoxidation of cyclohexene, then the ring-open reaction to afford 1,2cyclohexanediol. ${ }^{9}$

However, in both examples, excess formic acid (10 equiv.) was used as both reagent and solvent. Bases such as sodium hydroxide ( $3 \mathrm{~N}$ aqueous solution; 2 equiv. of $\mathrm{NaOH}, 13 \mathrm{~N}$ aqueous solution ${ }^{9}$ ) were introduced to hydrolyze the epoxides and formate derivatives to form diols. Excessive use of caustic materials are hazardous and could negatively impact waste control. Interestingly, in Swern's report, “...during a period of several months exposure to the air the neutralization equivalent of hydroxy-formoxystearic acid diminished...These facts suggest the possibility of obtaining the free glycols from the formate esters by hydrolysis with water alone", they suggested that free diols can possibly be obtained by hydrolysis using water. ${ }^{8}$ Herein, we report the conversion of terminal and internal alkenes into diols using 1.06 equivalent of formic acid ( $5.3 \mathrm{mmol}), 1.05$ equivalent of hydrogen peroxide ( $5.2 \mathrm{mmol}$ ) and 1 equivalent of alkenes (5.0 $\mathrm{mmol}$ ), followed by hydrolysis of epoxides using only water. This method reduces the amount of formic acid and avoids production of heat from adding sodium hydroxide to hydrolyze hydroxyformoxy compounds. ${ }^{10}$ 
(1)

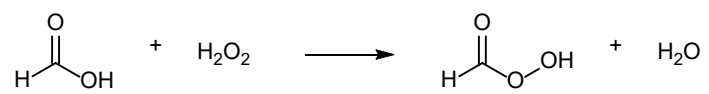

(2)<smiles>[R]C=C[CH]CC[Y]</smiles>

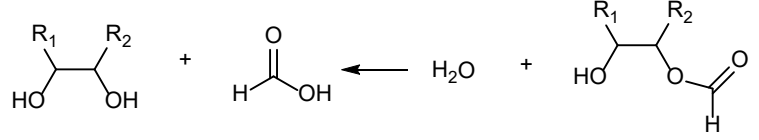

Scheme 1. Proposed reaction scheme by Swern's group. ${ }^{8}$

In this communication, we firstly carried out the dihydroxylation of cyclohexene under the following conditions: under nitrogen, cyclohexene ( $5.0 \mathrm{mmol}, 0.413 \mathrm{~g}$ ) and formic acid ( $5.3 \mathrm{mmol}, 0.244$ g) were mixed and hydrogen peroxide $(5.2 \mathrm{mmol}, 0.509 \mathrm{~g}, 35$ wt. $\% \mathrm{H}_{2} \mathrm{O}_{2}$ in water) was added dropwise into the same flask. After stirring for 5 minutes at room temperature, the reaction was gradually heated to $75^{\circ} \mathrm{C}$ and stirred for 3 hours. Using GCMS analysis, the epoxide species were detected along with the production of diols and hydroxy-formoxy compounds (See Supporting Information, Figure S1-S4 and Table S1). After the reaction was cooled to ambient temperature, an excess amount of water $(10 \mathrm{~g}, 556 \mathrm{mmol})$ was added and the reaction was heated to $75{ }^{\circ} \mathrm{C}$ for 6 hours to hydrolyze the hydroxy-formoxy compounds. The diol was initially extracted by dichloromethane and later obtained as the residue after evaporating solvents from the aqueous solution under reduced pressure. The recrystallized yield of 1,2 -cyclohexanediol is $39 \%$. In order to assure that hydroxy-formoxy species gets involved in water-driven hydrolysis, we investigated the conversion of hydroxy-formoxy compounds to diols using GC-MS. After the oxidation of cyclohexene using 1.06 equivalent of formic acid and 1.05 equivalent of hydrogen peroxide, acid and water were distilled and the residue was examined by GC-MS (See Supporting Information, Figure S9). Diols and hydroxy-formoxy compounds are the major products (diols: 58.572\%; hydroxy-formoxy compounds: $29.693 \%$ ). Then the crude was reconstituted in $10 \mathrm{~g}$ of water and heated to $75^{\circ} \mathrm{C}$ for 6 hours. Further GC-MS analysis of the hydrolyzed crude was taken after the complete removal of water and acid (See Supporting Information, Figure S10). The chromatogram displayed that the hydrolysis of hydroxy-formoxy compounds can be carried out with the presence of water (diols: 83.333\%; hydroxy-formoxy compounds: $1.870 \%$ ). According to the calibration curve, prior to water-driven hydrolysis, 2.478 mmol of 1,2-cyclohexanediol were present and after hydrolysis, a $25 \%$ increase was observed as $3.103 \mathrm{mmol}$ of 1,2-cyclohexediol (GC yield, 62\%) were present. This result successfully reflects Swern's observation (Scheme 1, eq. 3) pertaining to the waterdriven hydrolysis of hydroxy-formoxy compounds.

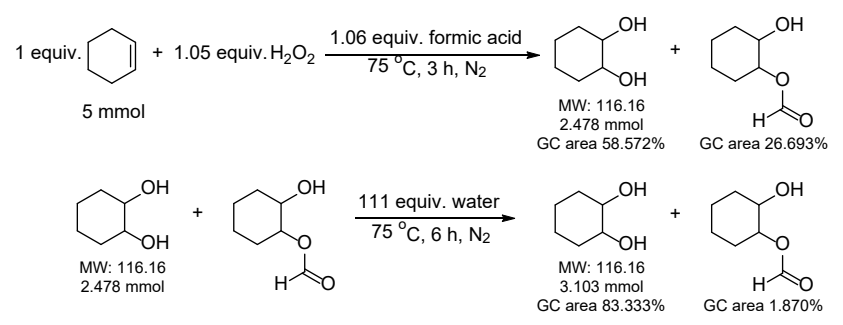

Scheme 2. Step-by-step GC-MS analysis of the dihydroxylation of cyclohexene.
In comparison with the previous report (Scheme 3) ${ }^{9}$, we simplify the procedure by using water as the hydrolyzing agent to avoid the use of excess bases. We further lower the usage of formic acid from 10 equivalent to 1.06 equivalent versus the alkene. Using this method, we can reduce cost and waste production at the same time. Indene is another internal alkene substrate that displays this efficient dihydroxylation under the same protocol with a decent recrystallized yield (53\%) (Table 1).

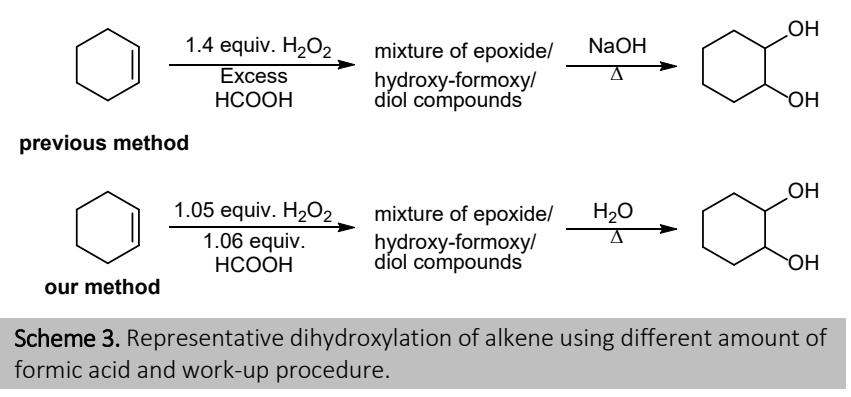

To broaden our scope, a series of terminal alkenes has been introduced to this report (Table 1.). Allylbenzene and styrene both led to diol derivatives 3 (43\%) and $\mathbf{4}$ (31\%).

Table 1. Dihydroxylation of terminal and internal alkenes. ${ }^{12}$

\begin{tabular}{|c|c|c|}
\hline 1 equiv. therernal alkenes/ & $\begin{array}{l}\frac{1.05 \text { equiv. } \mathrm{H}_{2} \mathrm{O}_{2}}{1.06 \text { equiv. } \mathrm{HCOOH}} \\
\quad \text { Neat, } 75^{\circ} \mathrm{C}, 3 \mathrm{~h}\end{array}$ & 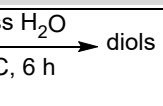 \\
\hline Substrate & Product & Yield (\%) \\
\hline Cyclohexene & 1,2-Cyclohexanediol 1 & $39 \%^{\mathrm{a}}(62 \%)^{\mathrm{b}}$ \\
\hline Indene & 1,2-Indanediol 2 & $53 \%^{a}$ \\
\hline Allylbenzene & Benzyl glycol 3 & $43 \%^{\mathrm{a}}$ \\
\hline Styrene & Styrene glycol 4 & $31 \%^{\mathrm{a}}$ \\
\hline
\end{tabular}

alsolated yield after recrystallization; ${ }^{b} \mathrm{GC}$ yield.

This method not only demonstrates a metal-free and base-free dihydroxylation of alkenes that leads to a cost-effective and ecofriendly diol production, but also has potential applicability to continuous flow systems for the large scale synthesis of valuable diols in the battery materials market. The application of continuous flow systems using this method is in due course.

\section{Funding Information}

Click here to insert sources of funding, grant numbers, etc. Do not repeat the same in the acknowledgment.

\section{Acknowledgment}

Argonne National Laboratory is a U. S. Department of Energy Office of Science laboratory operated by UChicago Argonne, LLC, under contract DE-AC02-06CH11357. The work published is sponsored by DOE EERE Vehicle Technology Office.

\section{Supporting Information}

YES (this text will be updated with links prior to publication)

\section{Primary Data}

NO (this text will be deleted prior to publication) 


\section{References and Notes}

(1) (a) Muschallik, L.; Molinnus, D.; Jablonski, M.; Kipp, C. R.; Bongaerts, J.; Pohl, M.; Wagner, T.; Schöning, M. J.; Selmer, T.; Siegert, P. RSC Adv. 2020, 10, 12206. (b) Ray, A. M.; Barbour, J. D.; McElfresh, J. S.; Moreira, J. A.; Swift, I.; Wright, I. M.; Žunič, A.; Mitchell, R. F.; Graham, E. E.; Alten, R. L.; Millar, J. G.; Hanks, L. M. J. Chem. Ecol. 2012, 38, 1151. (c) Ji, X.-J.; Huang, H.; Ouyang, P. K. Biotechnol. Adv. 2011, 29, 351.

(2) Fiume, M. M.; Bergfeld, W. F.; Belsito, D. V.; Hill, R. A.; Klaassen, C. D.; Liebler, D.; Marks, J. G.; Shank, R. C.; Slaga, T. J.; Snyder, P. W.; Andersen, F. A. Int. J. Toxicol. 2012, 31, 245S.

(3) Public Health Statement for Propylene Glycol; Agency for Toxic Substances \& Disease Registry, U.S. Department of Health and Human Services, Public Health Service: Atlanta, GA, 1997.

(4) Kitamura, T.; Inoue, Y.; Maeda, T.; Oyamada, J. Synth. Commun. 2016, 46, 39.

(5) Pyo, S.-H.; Hatti-Kaul, R. Adv. Synth. Catal. 2016, 358, 834.

(6) (a) Wang, C. Asian J. Org. Chem. 2018, 7, 509. (b) Plietker, B.; Niggemann, M.; Pollrich, A. Org. Biomol. Chem. 2004, 2, 1116. (c) Döbler, C.; Mehltretter, G. M.; Sundermeier, U.; Beller, M. J. Am. Chem. Soc. 2000, 122, 10289.

(7) Fringuelli, F.; Germani, R.; Pizzo, F.; Savelli, G. Synth. Commun 1989, 19, 1939.

(8) Swern, D.; Billen, G. N.; Findley, T. W.; Scanlan, J. T. J. Am. Chem. Soc. 1945, 67, 1786 .

(9) Roebuck, A.; Adkins, H. Org. Synth. 1955, 28, 35.

(10) In Ref. 8, the heat production was reported during the addition of sodium hydroxide into the mixture of the diol and hydroxyformoxy species.
(11) The experimental procedures and characterization data of 1,2 cyclohexanediol: under $\mathrm{N}_{2}$ atmosphere, $5 \mathrm{mmol}$ of cyclohexene $(0.413 \mathrm{~g})$ and $5.3 \mathrm{mmol}(0.244 \mathrm{~g})$ of formic acid were added, followed by dropwise addition of $5.2 \mathrm{mmol}$ of hydrogen peroxide ( $0.509 \mathrm{~g}, 35 \mathrm{wt} \% \mathrm{H}_{2} \mathrm{O}_{2}$ in water) into a 3-necked, $50 \mathrm{~mL}$ round bottom flask with a stir bar. After stirring at ambient temperature for 5 minutes, the reaction was gradually heated to $75^{\circ} \mathrm{C}$ for 3 hours (the reaction completion was monitored by GC-MS). After the reaction was cooled to ambient temperature, D.I. water (10 $\mathrm{mL}$ ) was added into the flask and the reaction was stirred for 6 more hours at $75{ }^{\circ} \mathrm{C}$. The crude 1,2-cyclohexanediol was extracted by $10 \mathrm{~mL} \times 3$ dichloromethane from the aqueous solution (the aqueous solution was kept for later use), followed by dehydration with $\mathrm{MgSO}_{4}$ anhydrous. After removing the $\mathrm{MgSO}_{4}$ and collecting the filtrate, the diol was obtained by evaporating solvent. To obtain more product, the aqueous solution was distilled to remove water and acid $\left(50 \mathrm{mbar}, 50^{\circ} \mathrm{C}\right)$, and the diol was collected as the residue. Further purification was performed via recrystallization (hexane/ethyl acetate, hexane/dichloromethane at $6{ }^{\circ} \mathrm{C}$ ). $127 \mathrm{mg}$ of 1,2-cyclohexanediol was obtained after the recrystallization as white crystals (39\% isolated yield). ${ }^{1} \mathrm{H}-\mathrm{NMR}\left(80 \mathrm{MHz}, \mathrm{CDCl}_{3}\right): \delta=$ 3.2-3.5 (m, 2H), 2.26 (br, 2H), $1.97(\mathrm{~m}, 2 \mathrm{H}), 1.71(\mathrm{~m}, 2 \mathrm{H}), 1.25(\mathrm{~m}$, $4 \mathrm{H}) .{ }^{13} \mathrm{C}-\mathrm{NMR}\left(20 \mathrm{MHz}, \mathrm{CDCl}_{3}\right): \delta=75.6,32.8,24.3$. EI-MS: $[\mathrm{M}]^{+}=$ 116.1 .

(12) This series of reactions was not optimized and the oily crude diols resulted in low isolated yields. 